\title{
In cross-sectional observations, dietary quality is not associated with CVD risk in women; in men the positive association is accounted for by BMI
}

\author{
Alexis C. Frazier-Wood ${ }^{1 *}$, Jihye $\mathrm{Kim}^{2} \dagger$, Jennifer S. Davis ${ }^{2}$, Su Yon Jung ${ }^{2}$ and Shine Chang $^{2}$ \\ ${ }^{1}$ Baylor College of Medicine, USDA/ARS Children's Nutrition Research Center, 1100 Bates Street, Houston, TX 77030, USA \\ ${ }^{2}$ Division of Cancer Prevention and Population Sciences, Department of Epidemiology, University of Texas MD Anderson \\ Cancer Center, Houston, TX, USA
}

(Submitted 10 July 2014 - Final revision received 3 December 2014 - Accepted 7 January 2015 - First published online 27 March 2015)

\begin{abstract}
The role that BMI plays in the association between dietary quality and CVD risk is not known. We aimed to better understand this relationship using statistical methods which correct for sex-specific underreporting of dietary intake. Overall, dietary quality was assessed using the Healthy Eating Index (HEI) on data from 9797 non-pregnant adults (aged $>20$ years) who participated in the National Health and Nutrition Examination Survey from 2005 to 2010. CVD risk factors included blood pressure, fasting glucose and insulin, homeostatic models of insulin resistance (HOMA-IR), HDL- and LDL-cholesterol (HDL-C and LDL-C), TAG and C-reactive protein (CRP). We controlled for demographic and lifestyle covariates, and we used the population ratio approach (which adjusts for the underreporting of intake) to compare mean HEI scores between the top and bottom quartiles of covariate-adjusted CVD risk factors. In women, the total HEI score was not associated with any CVD risk factors (all $Q>0 \cdot 11$ ). In men, the total HEI score was associated with covariate-adjusted residuals for fasting insulin $(Q<0 \cdot 001)$, HOMA-IR $(Q<0 \cdot 001)$, HDL-C $(Q=0 \cdot 01)$, TAG $(Q=0.03)$ and CRP $(Q<0 \cdot 001)$. When we additionally adjusted for BMI, the association with total HEI score was not significant (all $P>0 \cdot 10$ ). In the present analyses, dietary quality was associated with five CVD risk factors in a sex-specific manner. Moreover, the association of BMI with CVD risk attenuated the relationship between CVD risk and diet, which suggests that BMI is an important factor in heart disease prevention.
\end{abstract}

Key words: Diet quality: Healthy Eating Index: CVD risk factors: The National Health and Nutrition Examination Survey: BMI

CVD was the leading cause of death in the United States in $2011^{(1)}$. Several metabolic risk factors are known to increase the risk of CVD both independently and synergistically ${ }^{(2,3)}$. These include hypertension $^{(4,5)}$, hypertriglyceridemia ${ }^{(6,7)}$, insulin resistance (marked by hyperglycemia and hyperinsulinemia) ${ }^{(8,9)}$, lowered HDL-cholesterol (HDL-C) ${ }^{(10,11)}$, increased LDL-cholesterol $(\mathrm{LDL}-\mathrm{C})^{(12,13)}$, increased C-reactive protein (CRP) levels ${ }^{(14)}$ and obesity (particularly abdominal obesity manifesting as a larger waist circumference; WC) ${ }^{(15-17)}$.

The etiology of CVD and its risk factors is multifactorial and comprises genetic, behavioural, psychosocial and nutritional components $^{(3)}$. In recognition of the importance of overall diet in the prevention of chronic diseases, including CVD, dietary guidelines in several countries have been developed for the prevention of chronic diseases (e.g. American Diabetes Association $^{(18)}$, Lichtenstein et $a l^{(19)}$, Guenther et $a l .{ }^{(20)}$, Peterkin ${ }^{(21)}$, McGuire et al. ${ }^{(22)}$, Becker et al. ${ }^{(23)}$, National
Health and Medical Research Council $\left.{ }^{(24)}\right)$. These guidelines make recommendations regarding both the quality and variety of overall diet, rather than focusing solely on individual nutrients ${ }^{(25,26)}$. Although individual guidelines vary somewhat, the overall recommendations for health almost universally focus on a pattern of intake which emphasises vegetables and fruits, whole grains, low-fat dairy, nuts, seeds, fish and unsaturated fatty acids ${ }^{(3,19,22)}$. The Dietary Guidelines for Americans are released every 5 years by the US Department of Agriculture (USDA) in conjunction with the US Department of Health and Human Services ${ }^{(22)}$. These guidelines encompass recommendations across twelve food group categories, including total fruits, whole fruits, total vegetables, greens and beans, whole grains, dairy, total protein foods, seafood and plant proteins, fatty acids, refined grains, $\mathrm{Na}$ and empty energy. To assess adherence to the current Dietary Guidelines for Americans, the USDA developed the Healthy Eating

\footnotetext{
Abbreviations: CRP, C-reactive protein; DBP, diastolic blood pressure; HDL-C, HDL-cholesterol; HEI, Healthy Eating Index; HEI-10, Healthy Eating Index2010; LDL-C, LDL-cholesterol; NHANES, National Health and Nutrition Examination Survey; PIR, poverty:income ratio; SBP, systolic blood pressure; USDA, US Department of Agriculture; WC, waist circumference.
}

*Corresponding author: Dr A. C. Frazier-Wood, fax +1 713798 7098, email lekki.wood@gmail.com

† Authors contributed equally. 
Index $(\mathrm{HEI})^{(20)}$. The HEI is a diet quality index that was most recently updated for the current Dietary Guidelines for Americans, which were released in 2010 (HEI-10). A high HEI score (indicating greater adherence to the Dietary Guidelines for Americans) has been associated with a small decrease in CVD incidence and mortality ${ }^{(27-30)}$ as well as a reduction in CVD risk as a result of lower blood pressure, increased HDL-C, decreased LDL-C, lower CRP levels and decreased fasting insulin ${ }^{(31,32)}$.

Despite the widespread adoption of diet quality indices to help prevent chronic disease, several deficiencies remain in our understanding of the association between diet quality and CVD risk factors. First, surveying large numbers of the population has necessitated the use of self-report dietary intake data. Self-report nutrition data are subject to underreporting for intake ${ }^{(33-35)}$, and the degree of underreporting can be sex specific ${ }^{(34)}$. Nutrient density models have been developed to correct dietary quality scores for underreporting by adjusting for overall reported intake ${ }^{(36)}$. These models employ energy-adjusted dietary quality, which does not show the same degree of underreporting that absolute dietary quality does ${ }^{(37)}$. However, nutrient density models have not been employed by previous research efforts on the association between the HEI and CVD risk factors, which has created the potential for biased parameter estimates and variability between studies due to methodological differences ${ }^{(36,38)}$. Second, some studies have employed intra-individual associations between diet quality and CVD risk factors when using 24-h recall data $^{(31)}$, which are best used at the group level, to aggregate out unrepresentative responses ${ }^{(39)}$. For this reason, nutrient density substitution models have been adapted for use in group comparisons in the population ratio approach $^{(38)}$. Finally, studies have not explicitly examined the extent to which BMI attenuates the relationship between diet quality and CVD risk. Although not all overweight or obese people have elevated CVD risk factors ${ }^{(40)}$, at the population level, obesity is a strong risk factor for CVD and BMI is strongly associated with all metabolic CVD risk factors ${ }^{(3,41-43)}$ as well as, to a moderate extent, with HEI-assessed diet quality $^{(31,44-46)}$. CVD risk, BMI and diet quality are all interrelated, but the reported associations between CVD risk and HEI scores have been small to moderate ${ }^{(27-29)}$, whereas the associations between obesity and both CVD and HEI have been stronger. We therefore hypothesised that the association between diet quality, as measured by the HEI, and CVD risk factors is partly mediated by BMI.

The goal of the present analyses was to use data from a large, nationally representative sample of the US population (National Health and Nutrition Examination Survey; NHANES), which was collected in three waves between 2005 and 2010, to examine whether any associations between total HEI-10 score and the CVD risk factors of hypertension, hyperlipidemia, glycemia measures, HDL-C, LDL-C and CRP were attenuated when controlling for the association between BMI or central adiposity (WC) and these CVD risk factors. In addition, we aimed to use a statistical approach which corrects for the underreporting of dietary intake at the group level, stratified by sex, to examine previously reported associations between CVD risk factors and dietary quality.

\section{Experimental methods}

\section{Population}

NHANES is an ongoing national survey conducted by the National Center for Health Statistics of the Centers for Disease Control and Prevention (CDC). NHANES is designed to assess the health and nutritional status of the US population by collecting a representative cross-sectional sample of the US non-institutionalised civilian population. The sampling methods have been described in detail elsewhere ${ }^{(47)}$; in brief, NHANES employed a complex multi-stage sampling approach based on the selection of counties, blocks, households and the number of people within a household. To correct for nonresponses and unequal probabilities for selection among the US ethnicities, the CDC has provided sampling weights for use in analysis.

We included data from three cycles of NHANES: 2005-6, 2007-8 and 2009-10 (n 31034 ). We included non-pregnant participants aged 20 years or older who (1) had completed the interview and the examination, (2) had provided 24-h in-person dietary data for at least the first day, (3) had data on BMI, (4) had examination data on at least one CVD risk factor (total $n$ 13828) and (5) had not specified their race as 'other' (due to small group size). This left us with a final sample of 13217 . We subsequently excluded individuals who were missing data on any of the covariates (age, ethnicity, education level, poverty:income ratio (PIR), smoking, alcohol use or physical activity for men and women, plus menopausal status for women), including the sampling weight (as was required by our analytic strategy), which left a total study population of 9797 (5250 men and 4547 women).

Those without information on fasting status, those without a value for the sample weight for fasting data, and those who did not report fasting for at least $8.5 \mathrm{~h}$ before attending the examination were excluded from analyses of outcomes of fasted CVD risk factors (5452 participants excluded; 4345 remaining). Participants excluded from the analysis of fasted outcomes were not different in terms of age, sex, ethnicity or education level than those who were included in the analysis of non-fasted outcomes $(P>0.05$; online supplementary Table S1).

\section{Measures}

Dietary intake. 24-h In-person dietary recall interviews were conducted at a mobile examination centre using a multi-pass technique over two separate days. All details of the dietary recall interview, including the multi-pass technique, are available in the NHANES dietary interviewers' procedures manual ${ }^{(48)}$. Methods for including more than one $24-\mathrm{h}$ recall within the population ratio method have not been validated for the $2010 \mathrm{HEI}$; therefore, only data from the first day 24-h recall were used in the present analyses ${ }^{(38)}$. 
Daily total energy intake ( $k c a l)$. Daily total energy intake (kcal) was provided by the CDC within the NHANES data. Total intake was calculated by converting reported food intake into macronutrient intake values for each food using the reported serving size and the USDA's Food and Nutrient Database for Dietary Studies ${ }^{(49)}$. The macronutrients were converted to energy using the following conversion factors: $16.7 \mathrm{~kJ} / \mathrm{g}(4 \mathrm{kcal} / \mathrm{g}$ ) for protein and carbohydrate and $37.7 \mathrm{~kJ} / \mathrm{g}$ $(9 \mathrm{kcal} / \mathrm{g})$ for total fat and saturated fat.

Dietary quality. Total HEI-10 score was used as a measure of dietary quality. Information on the HEI-2010 and its scoring method has been described elsewhere ${ }^{(20)}$, but in brief, total HEI-10 score is created from twelve dietary components, each of which reflected a discrete aspect of the Dietary Guidelines for Americans 2010 ${ }^{(22)}$ : total fruits, whole fruits, total vegetables, greens and beans, whole grains, dairy, total protein foods, seafood and plant proteins, fatty acids, refined grains, $\mathrm{Na}$ and empty energy. Each component has a minimum score of 0 and a maximum score between 5 and 20 . Each component is scored such that a higher value indicates better adherence to dietary guidelines. Details of the contribution of each food component to the total HEI-10 score is given in online supplementary Table S2. To partially control for the underreporting of diet intake, raw HEI scores were adjusted using the population ratio approach ${ }^{(38)}$. In brief, the population ratio is calculated using three steps: first, individual levels of food and nutrient intakes are calculated. Second, weighted means and a variance-covariance matrix of the four quartile residual groups used in the present analyses are generated. Using the means and the variancecovariance matrix, a Monte Carlo simulation dataset is generated to calculate standard errors of the HEI score in the group. Third, densities (per $4184 \mathrm{~kJ}(1000 \mathrm{kcal}))$ and percent (of total energy intake) are created using the simulation data. The population ratio approach calculates each component of the HEI-10 as a ratio of the population's energy total. The population ratio approach has been shown in computer simulations to give rise to the least biased content estimates from three approaches (the mean score, the mean ratio score and the population ratio), and it has been validated for use with the US population ${ }^{(38)}$. To create the total HEI-10 score, each component ratio score was summed. The total HEI score ranges from 0 to 100 , with higher scores indicating higher diet quality.

CVD risk factors. Systolic blood pressure (SBP) and diastolic blood pressure (DBP) were measured in a sitting position after $5 \mathrm{~min}$ of quiet rest. Certified physician examiners for blood pressure measured three consecutive readings of blood pressure. A fourth reading was recorded if any of the three measurements was interrupted or incomplete. For both SBP and DBP, we excluded participants who had a DBP reading of less than $30 \mathrm{mmHg}$ and used the average of all available readings. CRP concentrations were measured by high-sensitivity latex-enhanced nephelometry.

Fasting blood samples were collected during examination in the mobile examination centre for measures of glucose metabolism and lipid. Fasting glucose was measured using the hexokinase enzmatic assay; TAG were enzymatically measured with a series of coupled reactions; HDL-C was determined by using the direct HDL-C immunoassay method. NHANES does not provide information on equipment details or accuracy information for measures. All NHANES quality assurance and quality control protocols (QA/QC) meet the 1988 Clinical Laboratory Improvement Act mandates and are described in the NHANES Laboratory/Medical Technologies Procedures Manual (available at http://www.cdc. gov/nchs/data/nhanes/nhanes_09_10/lab.pdf).

Body weight, height and WC were measured and recorded by trained health technicians in the mobile examination centre. Participants were weighed in kg using a digital weight scale. Height was measured in a standing position using a stadiometer with a fixed vertical backboard. BMI was calculated as weight in $\mathrm{kg}$ divided by height in $\mathrm{m}^{2}$. The WC was measured at a point immediately above the iliac crest on the midaxillary line to the nearest $0 \cdot 1 \mathrm{~cm}$ at the end of normal expiration.

Demographic and health behaviour information. Demographic information was obtained via interviews in the home. Race/ethnicity was recorded as Mexican American, Other Hispanic, Non-Hispanic White or Non-Hispanic Black. Education level was recorded in three categories: lower than high school diploma, high school graduation, and higher than high school graduation. We categorised the family PIR into three categories ( $\geq 0.0$ to $1.3,>1.3$ to 3.5 and $>3.5$ ) according to Supplemental Nutrition Assistance Program eligibility. Smoking was categorised into three groups (never, former and current smokers) on the basis of two self-report questions on smoking status. Alcohol use was a categorised as yes/no, with 'yes' indicating at least twelve drinks in any 1 year. Physical activity was measured as the total metabolic equivalent of task-h/week, which were estimated from selfreported leisure-time activity level. For the 2005-6 data, we used the individual metabolic equivalent of task score for each leisure activity for calculation and summed values for all types of activities. Because the individual metabolic equivalent of task score was not available for the 2007-10 data, we calculated the individual metabolic equivalent of task scores using 4.0 and 8.0 for moderate and vigorous leisure-time physical activities, respectively ${ }^{(50)}$. Menopausal status (yes/no) was defined using three questions about the presence or absence of periods in past 1 year, the reason for amenorrhea and whether both ovaries were removed.

\section{Analyses}

All analyses were conducted in SAS version 9.3 (SAS Institute, Inc.). As described earlier, the population ratio approach was used to reduce bias in the estimate of the HEI-10 score ${ }^{(38)}$. The population ratio approach necessitates the use of means comparisons between groups, and it is only suitable for use in univariate analyses ( $t$ test comparisons). However, there are well-established risk factors that are associated with both low-dietary quality and raised BMI, which could confound potential associations between BMI and dietary quality. Therefore, we took the following analytic steps, which are commonly used to control for covariates in univariate analyses (e.g. Li et al. ${ }^{(51)}$ ), to determine whether the total HEI-10 score 
Table 1. Demographic characteristics, lifestyle variables, smoking, alcohol use and CVD risk factors in the NHANES (National Health and Nutrition Examination Survey) study population between 2005 and 2010

(Mean values and standard deviations; number of participants and percentages)

\begin{tabular}{|c|c|c|c|c|c|c|c|c|c|c|c|}
\hline & \multirow[b]{3}{*}{$n$} & \multicolumn{5}{|c|}{ Men ( $n$ 5250) } & \multicolumn{5}{|c|}{ Women ( $n$ 4547) } \\
\hline & & \multicolumn{2}{|c|}{$\begin{array}{l}\text { Normal weight* } \\
\quad(n \text { 1341) }\end{array}$} & \multicolumn{2}{|c|}{$\begin{array}{l}\text { Overweight/obese* } \\
\qquad(n \text { 3909) }\end{array}$} & \multirow[b]{2}{*}{$P \dagger$} & \multicolumn{2}{|c|}{$\begin{array}{l}\text { Normal weight* } \\
\quad(n \text { 1303) }\end{array}$} & \multicolumn{2}{|c|}{$\begin{array}{l}\text { Overweight/obese }{ }^{*} \\
\qquad(n 3244)\end{array}$} & \multirow[b]{2}{*}{$P \dagger$} \\
\hline & & Mean & SD & Mean & SD & & Mean & SD & Mean & SD & \\
\hline \multicolumn{12}{|l|}{ Demographic characteristics } \\
\hline Age (years) & 9797 & 40.7 & $16 \cdot 15$ & 46.9 & 14.94 & $<0.0001$ & $45 \cdot 8$ & $16 \cdot 06$ & $50 \cdot 0$ & $15 \cdot 24$ & $<0.0001$ \\
\hline Race/ethnicity (\%) & 9797 & & & & & 0.01 & & & & & $<0.0001$ \\
\hline NH White & & \multicolumn{2}{|c|}{$76 \cdot 6$} & \multicolumn{2}{|c|}{$76 \cdot 1$} & & \multicolumn{2}{|c|}{$84 \cdot 7$} & \multicolumn{2}{|c|}{$72 \cdot 8$} & \\
\hline NH Black & & \multirow{2}{*}{\multicolumn{2}{|c|}{$\begin{array}{c}11.9 \\
7\end{array}$}} & \multicolumn{2}{|c|}{$10 \cdot 1$} & & \multirow{2}{*}{\multicolumn{2}{|c|}{$\begin{array}{l}6.9 \\
4.8\end{array}$}} & \multicolumn{2}{|c|}{$14 \cdot 3$} & \\
\hline Mexican American & & & & \multirow{2}{*}{\multicolumn{2}{|c|}{$\begin{array}{l}9.5 \\
4.3\end{array}$}} & & & & \multirow{2}{*}{\multicolumn{2}{|c|}{$\begin{array}{c}8 \\
4.9\end{array}$}} & \\
\hline Other Hispanic & & \multicolumn{2}{|c|}{4.6} & & & & \multicolumn{2}{|c|}{3.5} & & & \\
\hline Education (\%) & 9797 & & & & & 0.29 & & & & & $<0.0001$ \\
\hline Up to twelve grade & & & & & & & & & & & \\
\hline High school & & & & & & & & & & & \\
\hline College or above & & & & & & & & & & & \\
\hline $\operatorname{PIR}(\%)$ & 9797 & & & & & $<0.0001$ & & & & & $<0.0001$ \\
\hline$\leq 1.3$ & & & & & & & & & & & \\
\hline$>1.3$ to $\leq 3.5$ & & & & & & & & & & & \\
\hline$>3.5$ & & & & & & & & & & & \\
\hline Lifestyle variables & & & & & & & & & & & \\
\hline Smoking (\%) & 9797 & & & & & $<0.0001$ & & & & & 0.001 \\
\hline Current & & & & & & & & & & & \\
\hline Former & & & & & & & & & & & \\
\hline Never & & & & & & & & & & & \\
\hline Alcohol use (\% yes) & 9797 & & & & & 0.4 & & & & & $<0.0001$ \\
\hline Total energy intake & 9797 & & & & & $<0.0001$ & & & & & 0.01 \\
\hline kcal & & 11340.5 & $4526 \cdot 35$ & 10444.4 & $3709 \cdot 39$ & & $7589 \cdot 3$ & 2511.52 & $7320 \cdot 5$ & 2535.94 & \\
\hline $\mathrm{kJ}$ & & $47448 \cdot 7$ & 18938.2 & 43699.4 & $15520 \cdot 1$ & & 31753.6 & 10508.2 & 30628.9 & $10610 \cdot 4$ & \\
\hline PA (MET) & 9797 & $26 \cdot 7$ & 43.72 & $19 \cdot 1$ & 30.62 & 0.0007 & $17 \cdot 1$ & 28.43 & 9.86 & 17.99 & $<0.0001$ \\
\hline Menopausal status (\% yes) & 4547 & & & & & & $47 \cdot 7$ & & 61.4 & & $<0.0001$ \\
\hline HEI-10 (population ratio adjusted) $\ddagger$ & 9797 & $52 \cdot 8$ & 1.31 & 52.7 & 0.62 & 0.95 & $60 \cdot 3$ & $1 \cdot 12$ & $57 \cdot 3$ & 0.93 & 0.04 \\
\hline HEI-10 (raw scores) & 9797 & $46 \cdot 3$ & 0.66 & $46 \cdot 8$ & 0.38 & 0.6404 & 50.9 & 0.66 & 48.8 & 0.52 & 0.0114 \\
\hline $\mathrm{BMI}\left(\mathrm{kg} / \mathrm{m}^{2}\right)$ & 9797 & 22.5 & 1.86 & 30.9 & $5 \cdot 18$ & $<0.0001$ & 22.0 & 2.05 & 32.5 & $6 \cdot 32$ & $<0.0001$ \\
\hline WC (cm) & 9797 & $85 \cdot 1$ & 7.34 & $107 \cdot 1$ & 13.29 & $<0.0001$ & $80 \cdot 1$ & 7.06 & $104 \cdot 1$ & $13 \cdot 83$ & $<0.0001$ \\
\hline CVD risk factors & & & & & & & & & & & \\
\hline $\mathrm{SBP}(\mathrm{mmHg})$ & 9669 & $119 \cdot 7$ & $15 \cdot 39$ & $124 \cdot 3$ & 14.66 & $<0.0001$ & $115 \cdot 5$ & $17 \cdot 44$ & $121 \cdot 6$ & $17 \cdot 36$ & $<0.0001$ \\
\hline $\mathrm{DBP}(\mathrm{mmHg})$ & 9635 & 69.4 & $11 \cdot 28$ & 73.4 & $11 \cdot 28$ & $<0.0001$ & $68 \cdot 6$ & $10 \cdot 10$ & $70 \cdot 2$ & 11.09 & 0.02 \\
\hline Fasting glucose $(\mathrm{mmol} / \mathrm{l})$ & 4345 & 5.58 & $1 \cdot 20$ & 6.04 & $1 \cdot 70$ & $<0.0001$ & $5 \cdot 24$ & 0.96 & 5.85 & 1.61 & $<0.0001$ \\
\hline Fasting insulin $(\mu \mathrm{U} / \mathrm{ml})$ & 4293 & 6.7 & 5.39 & $15 \cdot 2$ & 13.66 & $<0.0001$ & $6 \cdot 7$ & $4 \cdot 17$ & 14.5 & $10 \cdot 49$ & $<0.0001$ \\
\hline HOMA-IR & 4293 & 1.7 & 1.82 & $4 \cdot 2$ & 5.03 & $<0.0001$ & 1.6 & $1 \cdot 15$ & 3.9 & 3.66 & $<0.0001$ \\
\hline $\mathrm{HDL}-\mathrm{C}(\mathrm{mmol} / \mathrm{l})$ & 4319 & 1.45 & 0.40 & 1.20 & 0.32 & $<0.0001$ & 1.74 & 0.42 & 1.43 & 0.38 & $<0.0001$ \\
\hline TAG (mmol/l) & 4319 & $1 \cdot 21$ & 0.72 & 1.78 & 1.53 & $<0.0001$ & 1.02 & 0.54 & 1.60 & 1.35 & $<0.0001$ \\
\hline LDL-C (mmol/l) & 4228 & 2.84 & 0.94 & 3.11 & 0.89 & $<0.0001$ & 2.89 & 0.87 & 3.11 & 0.91 & 0.0002 \\
\hline CRP (mg/l) & 9471 & $2 \cdot 0$ & $8 \cdot 1$ & 4.0 & $7 \cdot 7$ & 0.002 & 2.0 & 6.5 & 6.0 & $7 \cdot 2$ & $<0.0001$ \\
\hline
\end{tabular}

$\mathrm{NH}$, non-Hispanic; PIR, poverty:income ratio; PA, physical activity; MET, metabolic equivalents; HEI-10, Healthy Eating Index-2010; WC, waist circumference; SBP, systolic blood pressure; DBP, diastolic blood pressure; HOMA-IR, homeostatic model of insulin resistance; HDL-C, HDL-cholesterol; LDL-C, LDL-cholesterol; CRP, C-reactive protein

* Normal weight was defined as BMI $<25 \mathrm{~kg} / \mathrm{m}^{2}$; overweight/obese as $\mathrm{BMI} \geq 25.0\left(\mathrm{~kg} / \mathrm{m}^{2}\right)$.

$\dagger$ From a $t$ test (continuous variables) or $\chi^{2}$ (categorical variables) test of mean differences within sex. 
differed for CVD risk factors and whether any associations remained after controlling for BMI in sex-stratified analyses.

(1) First, each risk factor was individually regressed for the covariates (ethnicity, education level, PIR, smoking, alcohol use, physical activity and menopausal status within women) using the model in equation 1 :

$$
\begin{aligned}
y_{i}= & \text { covariate }_{1 i}+\text { covariate }_{2 i}+\ldots \text { covariate }_{n i} \\
& +e_{i}^{\prime},(\text { equation } 1)
\end{aligned}
$$

where $y_{i}$ refers to a CVD risk factor for the $i$ th individual; covariate $_{1 i}$ refers to a covariate for the $i$ th individual; covariate ${ }_{2 i}$ refers to another covariate for the $i$ th individual; covariate ${ }_{n i}$ indicates all covariates that were used in a similar manner for the $i$ th individual; and $e_{i}$ refers to the residuals of the CVD factor, which is independent of the covariates. Thus, the residuals represent the proportion of variance in the CVD risk factors that cannot be explained by the covariates. Subsequently, the residuals were used as covariate-independent CVD risk factors.

(2) The residuals (covariate-independent CVD risk factors for women) were divided into quartiles.

(3) The HEI-10 score was devised for each quartile using the population ratio approach ${ }^{(38)}$.

(4) A $t$ test was conducted between the top and bottom quartiles, and the probability of difference was calculated under the normal distribution.

(5) For each CVD risk factor that showed significant differences in HEI-10 score between the top and bottom quartiles, the risk factor was then regressed for the covariates (ethnicity, education level, PIR, smoking, alcohol use, physical activity, menopausal status and BMI within women) using the linear regression model specified in equation 2 :

$$
\begin{aligned}
y_{i}= & \mathrm{BMI}_{i}+\text { covariate }_{1 i}+\text { covariate }_{2 i}+\ldots \text { covariate }_{n i} \\
& +f_{i}^{\prime},(\text { equation } 2)
\end{aligned}
$$

where $\mathrm{BMI}_{i}$ refers to the $\mathrm{BMI}$ for the $i$ th individual and $f_{i}^{*}$ refers to the residuals of the CVD risk factor, which are independent of the covariates and BMI. Thus, the residuals for this step represent the proportion of variance in the CVD risk factors that cannot be explained by the covariates or BMI. Subsequently, these residuals were used as BMI and covariate-independent CVD risk factors.

(6) The residuals (BMI- and covariate-independent CVD risk factors for women) were divided into quartiles.

(7) The HEI-10 score was devised for each quartile using the population ratio approach.

(8) A $t$ test was conducted between the top and bottom quartiles, and the probability of difference was calculated under the normal distribution.

The analyses were subsequently repeated for men (without controlling for menopausal status or hormone therapy use),

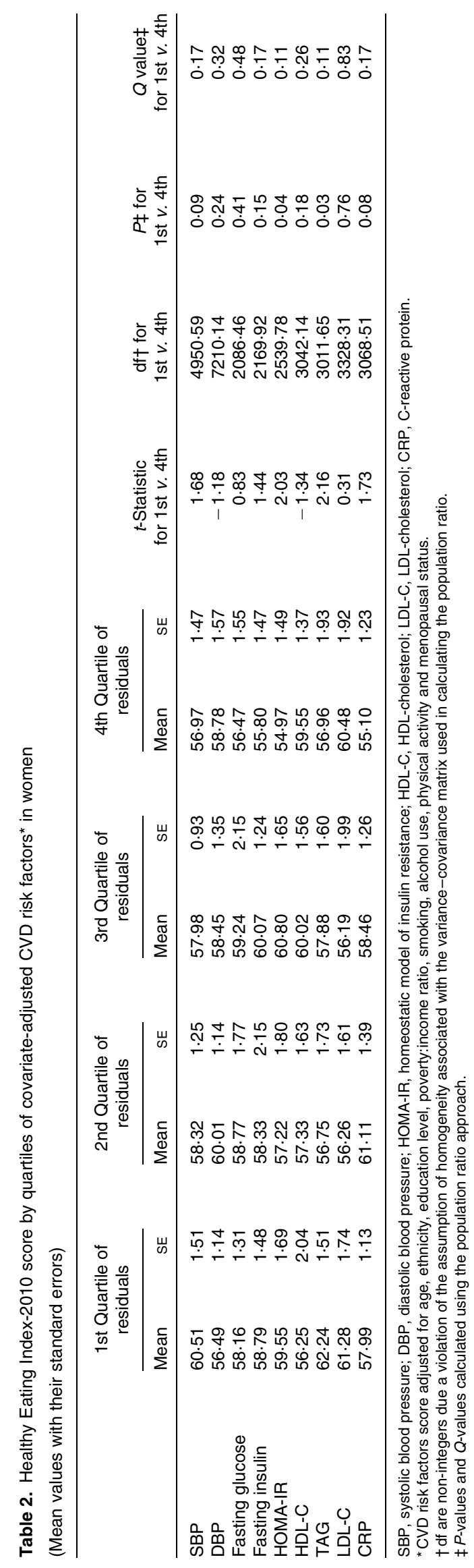


and the results are presented separately for men and women. A false discovery rate correction for multiple testing was applied to these analyses, and corrected $Q$-values were reported $^{(52)}$.

Because the population ratio approach is recommended by the National Cancer Institute (NCI), we focus on this methodology. We provide results obtained without applying the population ratio approach in online supplementary Tables $\mathrm{S} 3$ and $\mathrm{S} 4$.

\section{Results}

Demographic characteristics are presented in Table 1. Lean $v$. obese status was associated with age, ethnicity, PIR, smoking and physical activity in men and women (all $P=0.006$ to $<0.0001)$ as well as menopausal status in women $(P<0.0001)$. Education level was associated with lean $v$. obese status in women $(P<0 \cdot 0001)$ but not men $(P=0 \cdot 29)$, as was alcohol level $(P<0.0001$ and $P=0 \cdot 40$, respectively).

\section{Associations between Healthy Eating Index-2010 score and covariate-adjusted CVD risk factors using the population ratio approach}

When comparing the first and last quartiles of CVD risk factor for total HEI-10 score, dietary quality was not associated with the covariate-independent CVD risk factors $\operatorname{SBP}(Q=0 \cdot 17)$, DBP $(Q=0 \cdot 32)$, fasting glucose $(Q=0 \cdot 48)$, fasting insulin $(Q=0 \cdot 17)$, HOMA-IR $(Q=0 \cdot 11)$, HDL-C $(Q=0 \cdot 26)$, TAG $(Q=0 \cdot 11)$, LDL-C $(Q=0 \cdot 83)$ and CRP $(Q=0 \cdot 17)$ in women (Table 2 ). In men, dietary quality was associated with fasting insulin $(Q=0 \cdot 001)$, HOMA-IR $(Q=0 \cdot 001)$, HDL-C $(Q=0 \cdot 01)$, TAG $(Q=0.04)$ and CRP $(Q=0.01)$ but not with SBP $(Q=0 \cdot 19)$, DBP $(Q=0 \cdot 19)$, fasting glucose $(Q=0 \cdot 07)$ or LDL-C $(Q=0 \cdot 48$; Table 3).

\section{Associations between Healthy Eating Index-2010 score} and $B M I-$ and covariate-adjusted CVD risk factors using the population ratio approach

For those associations between HEI-10 and covariate-adjusted CVD risk factors, we additionally adjusted the risk factors for BMI. Subsequently, no associations between dietary quality and CVD risk factors remained statistically significant (all $Q>0 \cdot 10$; Table 4). We also ran the associations with CVD risk factors adjusted for covariates and WC (but not BMI), because central adiposity is a CVD risk factor. We observed the same pattern of results (online supplementary Table S5).

\section{Discussion}

The goal of the present study was to use a large, nationally representative US dataset (NHANES) to examine whether any observed associations between dietary quality, as measured by the HEI-10, and CVD risk factors were attenuated when the association between BMI and CVD risk factors was taken into account. To our knowledge, this is the first study to use the population ratio approach to examine the association

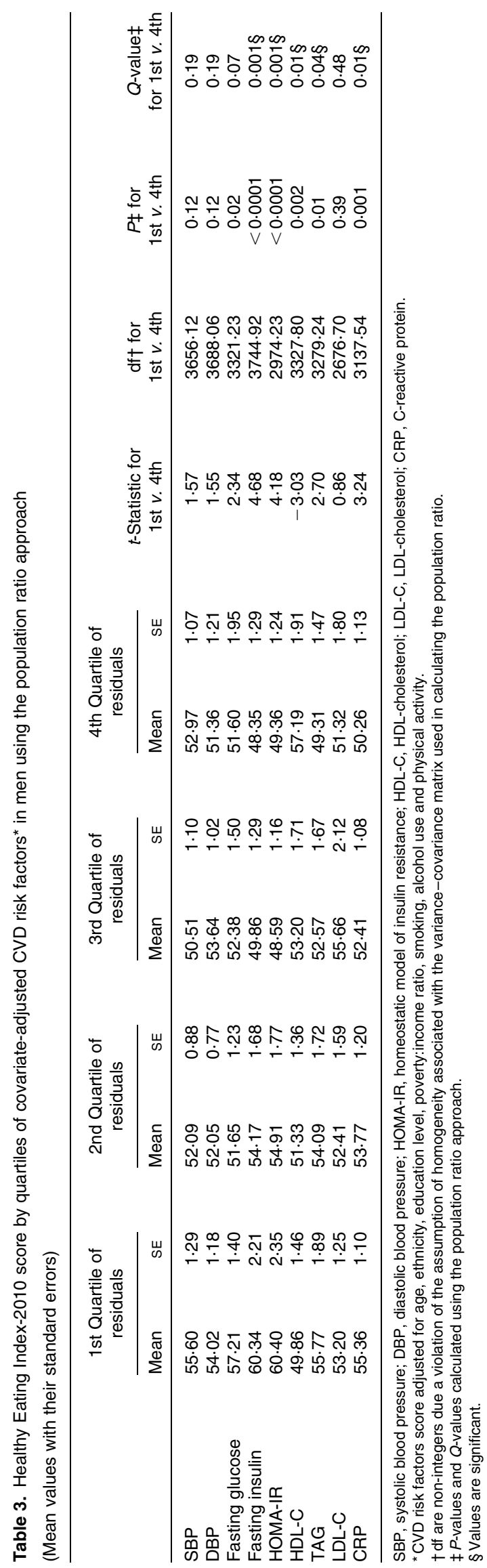


Table 4. Healthy Eating Index-2010 score by quartiles of BMI- and covariate-adjusted CVD risk factors* in men using the population ratio approach (Mean values with their standard errors)

\begin{tabular}{|c|c|c|c|c|c|c|c|c|c|c|}
\hline & \multicolumn{2}{|c|}{$\begin{array}{l}\text { 1st Quartile of } \\
\text { residuals }\end{array}$} & \multicolumn{2}{|c|}{$\begin{array}{l}\text { 2nd Quartile of } \\
\text { residuals }\end{array}$} & \multicolumn{2}{|c|}{$\begin{array}{l}\text { 3rd Quartile of } \\
\text { residuals }\end{array}$} & \multicolumn{2}{|c|}{$\begin{array}{l}\text { 4th Quartile of } \\
\text { residuals }\end{array}$} & \multirow{2}{*}{$\begin{array}{c}P \nmid \text { for } \\
\text { 1st v. 4th }\end{array}$} & \multirow{2}{*}{$\begin{array}{l}Q \text {-valuet fo } \\
\text { 1st v. 4th }\end{array}$} \\
\hline & Mean & SE & Mean & SE & Mean & SE & Mean & SE & & \\
\hline Fasting insulin & 54.52 & 1.78 & 53.51 & 1.97 & $54 \cdot 12$ & 1.84 & 49.52 & 1.08 & 0.0162 & 0.34 \\
\hline HOMA-IR & $54 \cdot 17$ & 1.83 & 53.34 & 1.45 & 52.73 & 1.68 & 51.66 & 1.50 & 0.2894 & 0.28 \\
\hline HDL-C & 51.32 & 1.59 & 51.06 & 1.60 & 53.91 & $1 \cdot 19$ & $55 \cdot 13$ & 2.00 & 0.1366 & 0.14 \\
\hline TAG & 53.41 & 1.40 & 55.01 & $2 \cdot 24$ & $52 \cdot 72$ & 1.55 & $50 \cdot 10$ & 1.52 & 0.1101 & $0 \cdot 10$ \\
\hline CRP & 51.80 & 1.00 & 53.96 & $1 \cdot 17$ & 53.68 & 0.93 & $51 \cdot 17$ & 1.31 & 0.7001 & 0.73 \\
\hline
\end{tabular}

HOMA-IR, homeostatic model of insulin resistance; HDL-C, HDL-cholesterol; CRP, C-reactive protein.

${ }^{*}$ CVD risk factors score adjusted for BMI, and age, ethnicity, education level, poverty:income ratio, smoking, alcohol use and physical activity

$\dagger P$ values and $Q$-values calculated using the population ratio approach.

between CVD risk factors and HEI-10 as well as the first study to stratify such analyses by sex. No significant associations between dietary quality and CVD risk factors were seen in women, but in men overall, dietary quality was significantly associated with five CVD risk factors. However, these associations were attenuated and no longer significant when we statistically controlled for the effect of BMI on CVD risk factors.

Previous research has shown that dietary quality, as measured by the HEI, shows a weak association with CVD incidence $^{(28,29)}$ and mortality ${ }^{(30)}$ in both men and women. It is therefore not surprising that other research has associated dietary quality with the present panel of CVD risk factors ${ }^{(31,32)}$. However, methodological advances in nutritional epidemiology since these studies were first published allow for confirmation of these findings. Such statistical improvements in the analysis address issues such as the use of intra-person analyses on 24-h recall data, which should be compared at the group level, and the difficulty in accounting for the underreporting of nutritional data, which varies by $\operatorname{sex}^{(34)}$. Specifically, the population ratio approach uses the ratio of nutrients to reported energy intake ${ }^{(38)}$, and it is a modification of nutrient density models which correct food frequency data for reported energy. For these reasons, the population ratio approach is suitable for $24-\mathrm{h}$ recall data ${ }^{(36)}$. Applying the population ratio approach resulted in small but important differences in the association between diet quality and CVD risk factors. For example, with the population ratio approach, HDL-C was not associated with dietary quality in women $(P=0 \cdot 12)$, but without the population ratio approach, they were associated $(P<0.05)$. Similarly, when applying the population ratio approach, fasting DBP was not associated with diet quality in men $(P=0 \cdot 30)$, but without that approach, they were associated ( $P=0.01 ; Q=0.04)$. The differences between the two approaches were not frequent enough and were not of a magnitude to draw consistent conclusions regarding the direction of effect for reducing bias in self-report nutrition data using the population ratio approach, and other studies should examine this in a more systematic manner. Using the population ratio approach, we report that HEI-10 score is associated with five known CVD risk factors: insulin, HOMA-IR, HDL-C, TAG and CRP in men. This confirms the well-known association between overall dietary quality (as defined by a number of indices) and numerous health indicators, including markers of inflammation, lipid parameters and insulin axis measures ${ }^{(31,53-55)}$

In the present analyses, HEI-10 score was not associated with blood pressure or LDL-C. Although this is contrary to one previous report that used NHANES data ${ }^{(31)}$, the present finding is in line with the larger body of literature that largely centres on reducing $\mathrm{Na}$ or adhering to the Dietary Approaches to Stop Hypertension diet ${ }^{(57,58)}$. This literature shows only a very moderate association between diet and blood pressure ${ }^{(56)}$. Because the HEI does not specifically measure Dietary Approaches to Stop Hypertension dietary recommendations, it is entirely plausible that HEI adherence is not associated with improved hypertension. Indeed, in the previous HEI/NHANES report, only SBP (not DBP) was significantly associated with overall HEI score, and it was only associated between the first and second quartiles of HEI intake, not overall ${ }^{(31)}$. This may highlight the importance of using statistical approaches designed for the type of nutritional data collected. The present study therefore adds weight to a growing body of literature which suggests that general diet quality recommendations are not associated with blood pressure.

It was surprising that no significant associations were observed between dietary quality and CVD risk factors in women, although we did find one report of a similar pattern of results with a French sample that also used the $\mathrm{HEI}^{(56)}$. Other lifestyle behaviours, such as screen time, show sexspecific associations with CVD risk factors ${ }^{(59,60)}$, and it has been suggested that other correlates of sex, such as smoking rates and alcohol intake, might account for differences, which would indicate a ceiling effect to CVD risk. This suggests that work needs to be done in this area with more careful matching between men and women on background characteristics. Furthermore, the need to conduct sex-stratified analyses on CVD risk is highlighted. If the results from the present study and those of Drewnowski et al. ${ }^{(56)}$ in France are replicated, there could be important public health implications for devising sex-sensitive strategies to prevent CVD risk. It is important that work is conducted to replicate the results in the present study and to explain the physiology underlying these findings.

For the first time, we show that associations between dietary quality and CVD risk factors are attenuated when accounting for the effect of BMI on CVD risk factors. Previous analyses 
have controlled for BMI; however, they have not explicitly compared associations between BMI-adjusted and BMI-unadjusted risk factors ${ }^{(31)}$. In the present study, the associations (all $Q<0.04$ ) were no longer significant after we controlled for BMI (all $Q \geq 0 \cdot 10$ ). Although the population ratio approach, which is necessary to achieve the most accurate analysis of 24-h self-report nutrition data, negates the possibility of an explicit comparison of effect sizes, as is the case with a Sobel test, it is clear that the associations are dramatically attenuated when controlling for BMI. Before controlling for BMI, there is an average of 9 HEI-10 points between the top and bottom quartiles for each significant risk factor. After controlling for BMI, this drops to a 3-2-point difference. This suggests that dietary quality may not be associated with CVD risk factors in men over and above the association of BMI with CVD risk factors. Whether this implies that BMI is a more suitable target for intervention than dietary quality when attempting to reduce CVD risk requires further analysis. We are not aware of any intervention trials which target diet quality and successfully modify CVD risk factors but examine the effects independent of BMI changes ${ }^{(61)}$. This is likely because BMI and central obesity are considered correlates of CVD risk. Although the findings in this study need replication and examination in interventions, at this stage the results suggest that BMI is a worthwhile target for reducing CVD risk.

There are clear limitations for the present study. First, its cross-sectional nature makes causal inferences and inferences regarding the effect of interventions on CVD risk factors impossible. Second, dietary data was self-reported, not measured, but it did directly follow the 24-h recall period. Although HEI is correlated with several biological markers of nutritional intake ${ }^{(62,63)}$, and although we employed a statistical approach that has been shown to minimise underreporting and bias in self-report dietary data ${ }^{(36,38)}$, it is likely that the present models subsume error and other sources of variability. As such, we focus on the overall patterns of results, and not on specific parameter estimates. Third, the HEI was not developed specifically for the prevention of CVD. Therefore, it is not a very sensitive measure of the potential for diet to affect CVD risk; for example, grains are not differentiated into refined and unrefined, which would show differential associations with CVD risk factors ${ }^{(26)}$. Finally, the HEI is only one measure of dietary quality that reflects adherence to the Dietary Guidelines for Americans; other measures should be explored for sex- or risk factor-specific associations.

Despite these limitations, we used the population ratio approach to examine whether dietary quality, as measured by overall HEI-10 score, is associated with CVD risk factors and whether any associations can be attributed to BMI. We corroborate previous findings that dietary quality is associated with HOMA-IR, insulin, HDL-C, TAG and CRP in men, but we cannot support its association with blood pressure and LDL-C. Furthermore, we provide the second report that HEI-assessed dietary quality is not associated with CVD risk factors in women ${ }^{(56)}$, which highlights a sex-specific association between dietary quality and CVD risk. Finally, we show that any significant associations in men are attenuated to non-significance when statistically controlling for the effect of BMI on CVD risk factors. In sum, the present study offers several insights for understanding the association between CVD risk and diet, including the importance of using careful statistical approaches in the analysis of dietary data and examining the potential for CVD risk factors to be sex specific. Furthermore, previous research has established that BMI is a key correlate of CVD risk, and the present study uniquely suggests that BMI is a CVD risk factor over and above dietary quality. We encourage future trials to examine whether this has clinical relevance by assessing the effect of intentionally reducing BMI on CVD risk factors without adding any further intervention aimed to improve dietary quality.

\section{Supplementary material}

To view supplementary material for this article, please visit http://dx.doi.org/10.1017/S0007114515000185

\section{Acknowledgements}

A. C. F.-W. is funded by the American Heart Association (grant no: 14BGIA18740011) and the USDA/ARS Children's Nutrition Research Center at Baylor College of Medicine. This work is a publication of the USDA/ARS Children's Nutrition Research Center, Department of Pediatrics, Baylor College of Medicine, Houston, TX, and it is funded in part with federal funds from the USDA/ARS under Cooperative Agreement No. 309-5-001058. The contents of this publication do not necessarily reflect the views or policies of the USDA, nor do the mention of trade names, commercial products or organisations imply endorsement from the US government. We thank Hwa Young for her assistance with data management and with statistical programming.

All authors report no conflicts of interest.

A. C. F.-W. formulated the research question, designed the analysis and wrote the article; J. K. conducted data analysis; J. K., J. S. D. and S. C. helped design the statistical analyses. In addition, all authors reviewed the final manuscript.

\section{References}

1. Hoyert DL \& Xu J (2012) Deaths: preliminary data for 2011. Natl Vital Stat Rep 61, 1-52.

2. Voight BF, Peloso GM, Orho-Melander M, et al. (2012) Plasma HDL cholesterol and risk of myocardial infarction: a Mendelian randomisation study. Lancet 380, 572-580.

3. Go AS, Mozaffarian D, Roger VL, et al. (2013) Heart disease and stroke statistics - 2013 update: a report from the American Heart Association. Circulation 127, e6-e245.

4. Thomas F, Rudnichi A, Bacri AM, et al. (2001) Cardiovascular mortality in hypertensive men according to presence of associated risk factors. Hypertension 37, 1256-1261.

5. Vasan RS, Larson MG, Leip EP, et al. (2001) Impact of highnormal blood pressure on the risk of cardiovascular disease. N Engl J Med 345, 1291-1297.

6. Sarwar N, Danesh J, Eiriksdottir G, et al. (2007) Triglycerides and the risk of coronary heart disease: 10,158 incident cases among 262,525 participants in 29 Western prospective studies. Circulation 115, 450-458. 
7. Cullen P (2000) Evidence that triglycerides are an independent coronary heart disease risk factor. Am J Cardiol 86, 943-949.

8. DeFronzo RA \& Ferrannini E (1991) Insulin resistance. A multifaceted syndrome responsible for NIDDM, obesity, hypertension, dyslipidemia, and atherosclerotic cardiovascular disease. Diabetes Care 14, 173-194.

9. Hanley AJG, Williams K, Stern MP, et al. (2002) Homeostasis model assessment of insulin resistance in relation to the incidence of cardiovascular disease: The San Antonio Heart Study. Diabetes Care 25, 1177-1184.

10. Cooney MT, Dudina A, De Bacquer D, et al. (2009) HDL cholesterol protects against cardiovascular disease in both genders, at all ages and at all levels of risk. Atherosclerosis 206, 611-616.

11. Asztalos BF, Collins D, Cupples LA, et al. (2005) Value of high-density lipoprotein (HDL) subpopulations in predicting recurrent cardiovascular events in the Veterans Affairs HDL Intervention Trial. Arterioscler Thromb Vasc Biol 25, 2185-2191.

12. Lu W, Resnick HE, Jablonski KA, et al. (2003) Non-HDL cholesterol as a predictor of cardiovascular disease in type 2 diabetes: The Strong Heart Study. Diabetes Care 26, 16-23.

13. Howard BV, Robbins DC, Sievers ML, et al. (2000) LDL cholesterol as a strong predictor of coronary heart disease in diabetic individuals with insulin resistance and low LDL: The Strong Heart Study. Arterioscler Thromb Vasc Biol 20, $830-835$.

14. De Ferranti SD \& Rifai N (2007) C-reactive protein: a nontraditional serum marker of cardiovascular risk. Cardiovasc Patbol 16, 14-21.

15. Wildman RP, Gu D, Reynolds K, et al. (2005) Are waist circumference and body mass index independently associated with cardiovascular disease risk in Chinese adults? Am J Clin Nutr 82, 1195-1202.

16. Wang Z \& Hoy WE (2004) Waist circumference, body mass index, hip circumference and waist-to-hip ratio as predictors of cardiovascular disease in Aboriginal people. Eur J Clin Nutr 58, 888-893.

17. Zhu S, Heshka S, Wang Z, et al. (2004) Combination of BMI and waist circumference for identifying cardiovascular risk factors in Whites. Obes Res 12, 633-645.

18. American Diabetes Association (2000) Nutrition recommendations and principles for people with diabetes mellitus. Diabetes Care 23, Suppl. 1, S43-S46.

19. Lichtenstein AH, Appel LJ, Brands M, et al. (2006) Summary of American Heart Association Diet and Lifestyle Recommendations revision 2006. Arterioscler Thromb Vasc Biol 26, 2186-2191.

20. Guenther PM, Casavale KO, Reedy J, et al. (2013) Update of the Healthy Eating Index: HEI-2010. J Acad Nutr Diet 113, 569-580.

21. Peterkin BB (1990) Dietary Guidelines for Americans, 1990 edition. J Am Diet Assoc 90, 1725-1727.

22. McGuire S, US Department of Agriculture \& US Department of Health and Human Services (2011) Dietary Guidelines for Americans, 2010. 7th ed. Washington, DC: US Government Printing Office. Adv Nutr 2, 293-294.

23. Becker W, Lyhne N, Pedersen N, et al. (2004) Nordic Nutrition Recommendations 2004 - integrating nutrition and physical activity. Food Nutr Res 48, 178-187.

24. National Health and Medical Research Council (2013) Australian Dietary Guidelines. Canberra: National Health and Medical Research Council.

25. Marshall S, Burrows T \& Collins CE (2014) Systematic review of diet quality indices and their associations with health-related outcomes in children and adolescents. $J$ Hum Nutr Diet 27, 577-598.

26. Wirt A \& Collins CE (2009) Diet quality - what is it and does it matter? Public Health Nutr 12, 2473-2492.

27. McCullough ML, Feskanich D, Stampfer MJ, et al. (2002) Diet quality and major chronic disease risk in men and women: moving toward improved dietary guidance. Am J Clin Nutr 76, 1261-1271

28. McCullough ML, Feskanich D, Stampfer MJ, et al. (2000) Adherence to the Dietary Guidelines for Americans and risk of major chronic disease in women. Am J Clin Nutr 72, 1214-1222.

29. McCullough ML, Feskanich D, Rimm EB, et al. (2000) Adherence to the Dietary Guidelines for Americans and risk of major chronic disease in men. Am J Clin Nutr 72, 1223-1231.

30. Reedy J, Krebs-Smith SM, Miller PE, et al. (2014) Higher diet quality is associated with decreased risk of all-cause, cardiovascular disease, and cancer mortality among older adults. J Nutr 144, 881-889.

31. Nicklas TA, O'Neil CE \& Fulgoni VL (2012) Diet quality is inversely related to cardiovascular risk factors in adults. J Nutr 142, 2112-2118.

32. Ford ES, Mokdad AH \& Liu S (2005) Healthy Eating Index and C-reactive protein concentration: findings from the National Health and Nutrition Examination Survey III, 1988-1994. Eur J Clin Nutr 59, 278-283.

33. Schoeller DA, Thomas D, Archer E, et al. (2013) Self-reportbased estimates of energy intake offer an inadequate basis for scientific conclusions. Am J Clin Nutr 97, 1413-1415.

34. Mertz W, Tsui JC, Judd JT, et al. (1991) What are people really eating? The relation between energy intake derived from estimated diet records and intake determined to maintain body weight. Am J Clin Nutr 54, 291-295.

35. Heitmann BL \& Lissner L (1995) Dietary underreporting by obese individuals - is it specific or non-specific? BMJ 311, 986-989.

36. Willett W \& Stampfer M (1998) Implications of total energy intake for epidemiologic analyses. In Nutritional Epidemiology, pp. 273-301 [W Willet, editor]. New York: Oxford University Press.

37. Voss S, Kroke A, Klipstein-Grobusch K, et al. (1998) Is macronutrient composition of dietary intake data affected by underreporting? Results from the EPIC-Potsdam Study. European Prospective Investigation into Cancer and Nutrition. Eur J Clin Nutr 52, 119-126.

38. Freedman LS, Guenther PM, Krebs-Smith SM, et al. (2008) A population's mean Healthy Eating Index-2005 scores are best estimated by the score of the population ratio when one 24-hour recall is available. J Nutr 138, 1725-1729.

39. Willett WC (1998) Nutritional Epidemiology. New York: Oxford University Press.

40. Calori G, Lattuada G, Piemonti L, et al. (2011) Prevalence, metabolic features, and prognosis of metabolically healthy obese Italian individuals: the Cremona Study. Diabetes Care 34, 210-215.

41. Malik S, Wong ND, Franklin SS, et al. (2004) Impact of the metabolic syndrome on mortality from coronary heart disease, cardiovascular disease, and all causes in United States adults. Circulation 110, 1245-1250.

42. Rutter MK, Meigs JB, Sullivan LM, et al. (2005) Insulin resistance, the metabolic syndrome, and incident cardiovascular events in the Framingham Offspring Study. Diabetes 54 , 3252-3257.

43. Krauss RM, Winston M, Fletcher BJ, et al. (1998) Obesity: impact on cardiovascular disease. Circulation 98, 1472-1476. 
44. Gao SK, Beresford SA, Frank LL, et al. (2008) Modifications to the Healthy Eating Index and its ability to predict obesity: the Multi-Ethnic Study of Atherosclerosis. Am J Clin Nutr 88, 64-69.

45. Guo X, Warden BA, Paeratakul S, et al. (2004) Healthy Eating Index and obesity. Eur J Clin Nutr 58, 1580-1586.

46. Ervin RB (2008) Healthy Eating Index scores among adults, 60 years of age and over, by sociodemographic and health characteristics: United States, 1999-2002. Adv Data 1-16.

47. NHANES (2014) About the National Health and Nutrition Examination Survey. http://www.cdc.gov/nchs/nhanes/about_nhanes. htm (accessed November 2014).

48. NHANES (2014) Dietary Interview Component. http://www. cdc.gov/nchs/data/nhanes/nhanes_03_04/dietary_03_04.pdf (accessed November 2014).

49. US Department of Agriculture \& Agricultural Research Service (2008) The USDA Food and Nutrient Database for Dietary Studies, 3.0 - Documentation and User Guide. Beltsville, MD: Beltsville Human Nutrition Research Center, Food Surveys Research Group. http://www.ars.usda.gov/ $\mathrm{ba} / \mathrm{bhnrc} / \mathrm{fsrg}$

50. Ainsworth BE, Haskell WL, Whitt MC, et al. (2000) Compendium of physical activities: an update of activity codes and MET intensities. Med Sci Sports Exerc 32, S498-S504.

51. Li N, van der Sijde MR, Lifelines Cohort Study Group, et al. (2014) Pleiotropic effects of lipid genes on plasma glucose, HbA1c and HOMA-IR levels. Diabetes 63, 3149-3158.

52. Benjamini Y \& Hochberg Y (1995) Controlling the false discovery rate: a practical and powerful approach to multiple testing. J R Stat SOC B 57, 289-300.

53. Obarzanek E, Sacks FM, Vollmer WM, et al. (2001) Effects on blood lipids of a blood pressure-lowering diet: the Dietary Approaches to Stop Hypertension (DASH) Trial. Am J Clin Nutr 74, 80-89.

54. Shah BS, Freeland-Graves JH, Cahill JM, et al. (2010) Diet quality as measured by the healthy eating index and the association with lipid profile in low-income women in early postpartum. J Am Diet Assoc 110, 274-279.

55. Fung TT, McCullough ML, Newby PK, et al. (2005) Dietquality scores and plasma concentrations of markers of inflammation and endothelial dysfunction. Am J Clin Nutr 82, 163-173.

56. Drewnowski A, Fiddler EC, Dauchet L, et al. (2009) Diet quality measures and cardiovascular risk factors in France: applying the Healthy Eating Index to the SU.VI.MAX Study. J Am Coll Nutr 28, 22-29.

57. Sacks FM, Svetkey LP, Vollmer WM, et al. (2001) Effects on blood pressure of reduced dietary sodium and the Dietary Approaches to Stop Hypertension (DASH) diet. DASH-Sodium Collaborative Research Group. $N$ Engl J Med 344, 3-10.

58. Bray GA, Vollmer WM, Sacks FM, et al. (2004) A further subgroup analysis of the effects of the DASH diet and three dietary sodium levels on blood pressure: results of the DASH-Sodium Trial. Am J Cardiol 94, 222-227.

59. Frazier-Wood AC, Borecki IB, Feitosa MF, et al. (2014) Sex-specific associations between screen time and lipoprotein subfractions. Int J Sport Nutr Exerc Metab $\mathbf{2 4}$ 59-69.

60. Wijndaele K, Healy GN, Dunstan DW, et al. (2010) Increased cardiometabolic risk is associated with increased TV viewing time. Med Sci Sports Exerc 42, 1511-1518.

61. Yu-Poth S, Zhao G, Etherton T, et al. (1999) Effects of the National Cholesterol Education Program's Step I and Step II dietary intervention programs on cardiovascular disease risk factors: a meta-analysis. Am J Clin Nutr 69 , 632-646.

62. Hann CS, Rock CL, King I, et al. (2001) Validation of the Healthy Eating Index with use of plasma biomarkers in a clinical sample of women. Am J Clin Nutr 74, 479-486.

63. Weinstein SJ, Vogt TM \& Gerrior SA (2004) Healthy Eating Index scores are associated with blood nutrient concentrations in the third National Health And Nutrition Examination Survey. J Am Diet Assoc 104, 576-584. 